\title{
What is the 'Cause' in Causal Decision Theory?
}

\author{
Christopher Hitchcock
}

Received: 23 January 2013/ Accepted: 23 January 2013/Published online: 19 March 2013

(C) Springer Science+Business Media Dordrecht 2013

\begin{abstract}
A simple counterfactual theory of causation fails because of problems with cases of preemption. This might lead us to expect that preemption will raise problems for counterfactual theories of other concepts that have a causal dimension. Indeed, examples are easy to find. But there is one case where we do not find this. Several versions of causal decision theory are formulated using counterfactuals. This might lead us to expect that these theories will yield the wrong recommendations in cases of preemption. But they do not. The explanation, I argue, is that the 'cause' that has been the target of counterfactual analyses is a specific relation, 'actual causation', that is not needed for prospective deliberation. A simple counterfactual theory of causation seems to capture the notion of cause needed for causal decision theory. This shows, in opposition to some critics, that counterfactual theories of causation are not barking up the wrong tree.
\end{abstract}

\section{Introduction}

This is a paper about a non-existent problem.

It is well-known that counterfactual theories of causation in the tradition of Lewis (1973) face serious problems with cases of preemption and overdetermination. And it is something of a platitude that causation is of interest to philosophers in part because it is involved in so many other concepts of interest. These two observations would lead us to expect that cases of preemption and overdetermination will cause problems for counterfactual analyses of philosophically interesting concepts quite generally. And indeed, examples are easy to find.

C. Hitchcock $(\bowtie)$

Division of Humanities and Social Sciences, California Institute of Technology, 101-40, Pasadena, CA 91125, USA

e-mail: cricky@caltech.edu 
There is, however, an interesting exception. Causal decision theory explicitly takes rational decision-making to be a causal notion. And, in at least one prominent formulation, it analyzes rational decision-making in terms of counterfactuals. This would lead us to expect that we can construct counterexamples to causal decision theory involving cases of preemption and overdetermination. But there are no such counterexamples.

Understanding why this is so will teach us something both about the nature of causation, and about the prospects for a counterfactual analysis of causation. The standard target of philosophical analysis, the one David Lewis was aiming for, is a special causal relation that has come to be called actual causation. But the 'cause' in causal decision theory is not actual causation. If causal decision theory deserves the name, it follows that there must be more to causation than actual causation. David Lewis and others have held that what is distinctive about the causal relation they were attempting to analyze is that it is singular: it involves particular events, involving particular people or objects in particular places at particular times. However, causal decision theory is also singular in precisely the same sense. What is distinctive about actual causation is rather that is retrospective: it involves a kind of reasoning backward from effects to their causes. By contrast, causal decision theory is prospective: it involves reasoning forward from causes to their effects.

This observation about causal decision theory helps us to disarm one kind of objection to counterfactual theories of causation. While preemption and overdetermination still present challenges for counterfactual theories of actual causation, there is at least one causal notion that counterfactual theories get exactly right. This should be enough to assure us that counterfactual theories are not just barking up the wrong tree.

\section{Counterfactuals and Causation}

According to a simple counterfactual theory of causation (hereafter SCT), believed by no one, if $C$ and $E$ are distinct, ${ }^{1}$ occurring events, then:

$C$ is a cause of $E$ iff:

if $C$ hadn't occurred, $E$ would not have occurred.

In order to have any hope, the counterfactuals occurring in this definition must not backtrack. Suppose that there is a drop in atmospheric pressure, and that I observe a corresponding drop in the height of a column of mercury in my barometer. Shortly after that, it starts raining cats and dogs. ${ }^{2}$ We do not want our account to tell us that the falling mercury caused the storm. Hence we do not want to reason that if the mercury had not fallen, there would not have been a drop in atmospheric pressure, and hence there would have been no storm. Such a counterfactual is said to 'backtrack', since the changes introduced by our contrary-to-fact supposition that the mercury does not fall are allowed to propagate backward in time to one of the

\footnotetext{
${ }^{1}$ See Lewis (1986a) for discussion of the notion of distinctness.

2 This example is originally due to Jeffrey (1969).
} 
causes of the falling mercury, the atmospheric pressure. Thus, in a non-backtracking counterfactual, effects will (typically) depend counterfactually on their causes, but not vice versa.

A counterfactual theory of causation need not be committed to the view that all natural language counterfactuals are of the non-backtracking variety. Indeed backtracking counterfactuals sound very natural when one changes the construction slightly: 'if the mercury had not fallen, then there would have to have been no drop in atmospheric pressure'. Lewis himself (e.g. in 1979) acknowledged that the evaluation of counterfactuals is highly context-sensitive, and required only that there be some kind of counterfactual or some context of evaluation where backtracking does not occur. It certainly seems possible for a speaker to convey to her audience that her counterfactuals are to be understood in a non-backtracking way, and to proceed to communicate effectively and unambiguously with counterfactual language.

A number of attempts have been made to develop a semantics for nonbacktracking counterfactuals. Lewis (1979) argues that we should evaluate them by considering possible worlds in which their antecedents are made true by minor miracles. For example, to evaluate a counterfactual in which we suppose that the mercury had not dropped, we consider a possible world just like our world except that the barometer miraculously malfunctions. Woodward (2003) argues that we should consider a world in which the column of mercury is prevented from dropping by an intervention, where an intervention is an exogenous causal process meeting a number of specific requirements. Albert (2000), Kutach (2003) and Loewer (2007) have suggested that we evaluate such counterfactuals by considering possible worlds containing a past that is like the actual past in important respects. Maudlin (2007) has argued that the direction of time is a basic feature of our universe and that counterfactuals are to be analyzed by supposing the universe to evolve forward from an antecedently specified state, but not backward. ${ }^{3}$ I intend my discussion of counterfactual theories of causation to be sufficiently inclusive that any account of causation in terms of counterfactuals as characterized in one of these ways could qualify.

\section{Preemption and Overdetermination}

Counterfactual theories of causation run into problems with cases of preemption and overdetermination. In the standard example, ${ }^{4}$ Suzy throws a rock at a bottle with sufficient force to break it; her rock hits the bottle and it breaks. Now imagine that Billy is also there, and (1) would have thrown his rock and smashed the bottle if Suzy hadn't; or (2) throws his rock a split second later, so his rock sails through the empty space where the bottle used to be; or (3) throws his rock at the same time, with sufficient force to break the bottle by itself, hitting the bottle at exactly the

\footnotetext{
3 Although Maudlin does not advocate an analysis of causation in terms of counterfactuals, as we shall see in Sect. 3 below.

${ }^{4}$ First appearing in print in Lewis (2000), but tracing to an earlier draft of Hall (2004).
} 
same time as Suzy's rock hits the bottle. In all three cases, we want to say that Suzy's throw caused the bottle to break. ${ }^{5}$ Yet it is false that if Suzy had not thrown, the bottle would not have broken. Case (1) is an example of early preemption; case (2) is an example of late preemption; and case (3) is an example of overdetermination. In cases (1) and (2), Suzy's throw preempts some other cause that would otherwise have shattered the bottle, while in case (3) the bottle's shattering is overdetermined by the two throws.

There have been a number of attempts to add bells and whistles to the simple counterfactual theory of causation, starting with Lewis's attempt to identify causation with chains of counterfactual dependence (Lewis 1973). If the bells and whistles are themselves formulated in terms of counterfactuals, then there is a sense in which the result would still deserve to be called a counterfactual theory of causation. I will not consider any of theories in detail here (see e.g. Paul 2009 for an overview of some of these accounts). However, I want to point to one feature that a number of such accounts have (e.g. Hitchcock 2001; Yablo 2002; Woodward 2003; Halpern and Pearl 2005). These approaches often require that we assess causation by looking for counterfactual dependence while certain additional events are held fixed. For example, in case (1), the bottle would not have shattered if Suzy had not thrown her rock, if we also hold fixed that Billy did not throw his rock. In case (2), the bottle would not have shattered if Suzy had not thrown her rock, if we also hold fixed that Billy's rock did not hit the bottle. These counterfactuals are unusual in that we hold fixed events that are causally downstream of the antecedent. For example, in case (1) Billy would have thrown his rock if Suzy had not. Nonetheless, we evaluate the counterfactual 'If Suzy had not thrown...' while holding fixed that Billy did not throw. I called these 'explicitly-non-foretracking' (ENF) counterfactuals (Hitchcock 2001). We saw in the previous section that the counterfactuals that figure in an account of causation do not 'backtrack'. These new counterfactuals do not fully 'foretrack' either.

I want to consider a fairly broad line of criticism of counterfactual theories, articulated in different ways in Maudlin (2004, section 1), and Moore (2009, 422). The criticism is that cases of preemption and overdetermination show that counterfactual theories of causation are barking up the wrong tree. Even if it is possible to produce a definition in terms of counterfactuals that reproduces our causal judgments, something important will have been lost in abandoning SCT. If causation just is counterfactual dependence, why is it such a complicated kind of counterfactual dependence? If SCT had been true, we would have a simple and elegant theory that would go a long way to explain our interest in causation. But if causation is extensionally equivalent to some complicated relation that we can build out of counterfactual parts, it is fair to ask what is so special about just this relation, as opposed to any other that we can build out of counterfactual parts? Moreover, even if such a definition is extensionally adequate, it hardly seems plausible as an account of our concept of causation if it makes no contact with our actual reasoning about causation. When we judge, in each of our cases, that Suzy's throw caused the bottle to shatter, do we evaluate the truth of some complicated counterfactual? Isn't it rather

\footnotetext{
5 Lewis $(1973,2000)$ himself professed agnosticism about the third case, but accepted the first two.
} 
that we know that Suzy's throw caused the bottle to shatter, because her rock hit it with sufficient force to shatter it, and nothing interfered with the rock's shattering the bottle (or something similar)? Doesn't it seem, in light of these examples, that counterfactual dependence is the wrong starting place for a theory of causation?

\section{Counterexamples Everywhere}

It is often said that the concept of causation is of great interest to philosophers, because it is implicated in so many other concepts of interest to philosophers. Here is a small sample, drawn from the introductions of anthologies on causation:

Causation, conditionals, explanation, confirmation, dispositions, and laws form a cluster of closely related topics in metaphysics, philosophy of language, and philosophy of science. In addition, causation plays an important role in connection with many problems in other areas of philosophy, especially philosophy of mind and epistemology. (Sosa and Tooley 1993, 1)

[C]ausation has come to play a central role in the conceptual toolkit of contemporary philosophy, invoked in the foundations of popular approaches to a very wide range of other philosophical topics. To mention a few examples, it is crucial to popular arguments for the identity of mind and brain, central to discussions of free will, action, and agency, and often invoked in criteria for realism about particular topics; and it underpins some accounts of epistemic reliability and argument to best explanation, and grounds wellknown accounts of many other matters, such as reference, perception, memory, and the direction of time. (Price and Corry 2007, 1-2)

Philosophical theories that appeal to causation span the spectrum: they are to be found in ethics, in epistemology, and in virtually every topic in metaphysics: free will, personal identity, time, universals, laws of nature, and so on. (Beebee et al. 2009, 2)

This suggests an easy recipe for generating publications in philosophy: (1) Find a counterfactual theory of some one of the concepts mentioned above. (2) Construct a counterexample involving preemption or overdetermination. (3) Publish. Voilà! Here are three examples from the literature.

\subsection{Malcolm on Memory}

Malcolm (1963) offers the following analysis of factual memory:

A person $B$, remembers that $p$ from a time, $t$, if and only if $B$ knows that $p$, and $B$ knew that $p$ at $\mathrm{t}$, and if $B$ had not known at $t$ that $p$ he would not now know that $p$ (236)

The final clause takes the form of a counterfactual conditional. Munsat $(1966,23)$ offers the following counterexample. Suppose that I accidentally kill a deer while driving at $t$. Let $p$ be the proposition 'I killed a deer while driving'. At a later time, I 
remember the event. However, in the intervening period, someone has informed me that I killed a deer. Thus, even if I had not been aware of the fact at the time, I would still know it now. Nonetheless, I still remember having killed the deer. This is an example of late preemption. Shope $(1978,405)$ points out that we can also get a counterexample if someone would have informed me that I had killed a deer if I hadn't realized it at the time. This is an example of early preemption.

\subsection{Frankfurt on Alternate Possibilities}

Frankfurt (1969) offers a well-known counterexample to the principle of alternate possibilities. According to this principle, a person is morally responsible for what she has done only if she could have done otherwise. Suppose that Black wants Jones to perform a certain action. Black is a brilliant neuroscientist, and is able to scan Jones' brain, and can even control those parts of her brain that are responsible for moving her muscles. However, Black prefers not to show his hand. He will wait to see if Jones' first forms the intention to perform the action on her own. Only if she does not do so will he intervene and make her perform the action. In fact, Jones forms the intention and performs the action, without Black having to interfere in any way. Frankfurt claims that Jones is morally responsible for her action, even though she could not have done otherwise. This is a case of early preemption. Jones' intention causes her to act, and preempts Black's interference. But if Jones had not formed the intention to act, she would have acted anyway.

To make the structure clearer, consider the conditional analysis of ability, articulated by Hume, but popular among compatibilists about free will (see, e.g. Hobart 1934). Here is Hume's formulation:

By liberty, then, we can only mean a power of acting or not acting, according to the determinations of the will; that is, if we choose to remain at rest, we may; if we choose to move, we also may. (Hume 1748, §VIII)

On this analysis, then, I have the ability to perform action $A$ just in case the following conditional is true: if I were to will (choose, wish, desire, etc.) to do $A$, then I would do $A$. Black had the ability to do otherwise, just in case she would have done otherwise, had she willed to do so.

What Frankfurt's example strictly shows (if we accept that Black is morally responsible for her action) is that the conjunction of the principle of alternative possibilities and the conditional analysis of ability is false. (Of course, it also follows from this that the conjunction of the principle of alternative possibilities and any strictly stronger account of ability —one that takes the relevant conditional to be at least necessary for ability_-is false.) That leaves us with the option of rejecting the principle of alternative possibilities, as Frankfurt advocates, or else rejecting the conditional analysis of ability (and any stronger account). Smith (2003) recommends the second line of response, and this is the response that is most closely analogous to rejecting counterfactual theories of causation on the basis of preemption examples. 


\subsection{Finkish Dispositions}

According to the conditional analysis of dispositions, an object is disposed to manifest property $M$ in response to stimulus $S$, just in case it would manifest property $M$ if it were exposed to stimulus $S$. Martin (1994) and Lewis (1997) have offered counterexamples to this analysis. Here is a slightly embellished version of Lewis's counterexample. A wizard owns a collection of antique soda bottles, of which he is very fond. The bottles are made of glass and hence very fragile; i.e. they are disposed to break if struck. However, if anything were to strike one of the bottles (such as a stone thrown by Billy or Suzy), the wizard would cast a spell making the bottle as hard as diamond, thus preventing it from breaking. In fact, none of the bottles are ever struck.

This example has the structure of an early preemption case. This can be a bit difficult to see. The wizard's spell seems to be preempted, but by what? The answer, I think, is that it is preempted by the absence of striking. If this absence did not occur, i.e. if one of the bottles is struck, the wizard would have cast his spell.

\subsection{Diagnosis and Repair}

While it is not central to the aims of the present paper to assess these counterexamples in detail, or to try to improve on the various analyses, the dialectic to this point does suggest a natural diagnosis and repair. The fact that the analyses are vulnerable to counterexamples involving preemption suggests that counterfactuals figuring in the analyses are improper substitutes for genuinely causal conditions. If we convert the counterfactuals to the corresponding causal claims, the problems should go away.

Malcolm's analysis of memory would be amended as follows:

A person $B$, remembers that $p$ from a time, $t$, if and only if $B$ knows that $p$, and $B$ knew that $p$ at $\mathrm{t}$, and $B$ 's knowing $p$ at $t$ is a cause of her knowing that $p$ now.

This seems more plausible, and it would eliminate Munsat's counterexample.

We could amend the conjunction of the principle of alternative possibilities with the conditional analysis of ability in the following way:

A person is morally responsible for her action only if her action was caused by her willing to perform it.

That would leave it open whether we have replaced the conditional analysis of ability with a causal analysis of ability, ${ }^{6}$ or whether we have rejected the principle of alternate possibilities in favor of the proposed causal condition.

Lewis (1997) proposes amending the conditional analysis of dispositions with an explicitly causal analysis. His proposal is:

\footnotetext{
${ }^{6}$ Smith (2003) has advocated an indirect version of this strategy. He suggests that we understood ability in terms of dispositions, which in turn are to be understood causally along roughly the lines described below.
} 
Something $x$ is disposed at time $t$ to give response $M$ to stimulus $S$ iff, for some intrinsic property $B$ that $x$ has at $t$, for some time $t^{\prime}$ after $t$, if $x$ were to undergo stimulus $S$ at time $t$ and retain property $B$ until $t^{\prime}, S$ and $x$ 's having of $B$ would jointly be [a complete] cause of $x$ 's giving response $M .^{7}$ (Lewis 1997, 157)

The new conditional is an ENF counterfactual of the sort we encountered in Sect. 3. The antecedent specifies that $x$ is exposed to $S$ at $t$, but it explicitly stipulates that some effects of this do not occur, namely any that would involve a change in the intrinsic property $B$. In our example, the counterfactual would be: if the bottle were struck, and its internal structure remained unchanged (e.g. by the wizard's spell).... This is very close to the type of counterfactual that I and others have suggested we use for evaluating causation in preemption cases. Specifically, if we want to assess the following causal claim:

The absence of striking is a cause of the bottle's being intact,

we should consider the counterfactual:

If the bottle were struck, but the wizard did not cast his spell, then the bottle would shatter.

This suggests that we might try this rather odd-looking causal claim as our analysis of the corresponding disposition. ${ }^{8}$ It is certainly simpler than Lewis's analysis. So far as I know, this analysis has not been tried.

These analyses may well run into further problems. For example, the revised theory of memory faces familiar problems from 'deviant causal chains'. Suppose I accidentally kill a deer while driving, and write about the event in my diary. Later I forget about the event. Then I read my diary, and come to know that I killed a deer earlier. My earlier state of knowledge caused my later state, but not in the right kind of way for remembering. But the simple repairs do seem to be improvements, and they can be motivated in a principled way.

\section{Causal Decision Theory}

Suppose that an agent is faced with a choice of several different possible actions $a_{l}$, $a_{2}, \ldots, a_{n}$. The outcomes the agent considers possible are $o_{1}, o_{2}, \ldots, o_{m}$. We assume these outcomes are distinguished sufficiently finely so as to capture any differences the agent cares about. We let $A_{i}$ be the proposition that the agent performs action $a_{i}$, and $O_{j}$ the proposition that outcome $o_{j}$ occurs. The desirability of various states of the world for the agent are given by the function $\mathcal{D}$. Let ' $S \square \rightarrow T$ ' be the nonbacktracking counterfactual conditional 'if $S$ were the case, then $T$ would be the case'. Then, according to causal decision theory (CDT), the causal expected utility, $\mathcal{U}$, of action $a_{i}$ is:

\footnotetext{
7 Lewis's definition requires that $S$ and $B$ be 'an $x$-complete' cause, but the technical details of this defined notion are not central here. I have also changed some of the symbols for consistency.

${ }^{8}$ Those who are uncomfortable with causation by omission might substitute: the bottle is intact (in part) because it has not been struck.
} 


$$
\mathcal{U}\left(a_{i}\right)=\Sigma_{\mathrm{j}} P\left(A_{i} \square \rightarrow O_{j}\right) \times \mathcal{D}\left(A_{i} \& O_{j}\right) .
$$

This is essentially the formulation given in Gibbard and Harper (1978), and used by Joyce (1999). There are alternative formulations; Lewis (1981) argues that the formulations are essentially equivalent. CDT then recommends that the agent perform the action that maximizes causal expected utility.

Causal decision theory is normally contrasted with evidential decision theory (EDT), which recommends that the agent maximize evidential expected utility $\mathcal{V}$ :

$$
\mathcal{V}\left(a_{i}\right)=\Sigma_{\mathrm{j}} P\left(O_{j} \mid A_{i}\right) \times \mathcal{D}\left(A_{i} \& O_{j}\right) .
$$

(The locus classicus is Jeffrey 1983.) The guiding intuition behind CDT is that one should act so as to realize actions that are causes of those outcomes that one finds desirable (and prevent those outcomes that one finds undesirable). By contrast, EDT seems to recommend realizing those actions that are merely correlated with desirable outcomes. For example, if we wish to avoid a storm, CDT does not recommend trying to raise the column of mercury in a barometer, since if $R$ is the proposition that the column of mercury rises, and $S$ is the proposition that a storm occurs, $P(R \square \rightarrow S)=P(\sim R \square \rightarrow S)$. Indeed, if there is a small cost involved in raising the column of mercury, CDT will recommend against doing so. By contrast, since $R$ and $S$ are negatively correlated in probability, EDT would seem to recommend trying to raise the column of mercury.

Now a defender of EDT has a ready response to this example. If the agent is careful to consider her actual actions - say heating the barometer, or putting it in a hyperbaric chamber-she will realize that these actions are not correlated with the occurrence of a storm. But probabilistic correlations pose more serious problems in cases of what have become called 'medical Newcomb problems'. Suppose that the agent is susceptible to migraine headaches, something she strongly dislikes. The agent also enjoys eating chocolate, but not to nearly the extent that she dislikes migraines. Letting $M$ assert the occurrence of a migraine, and $C$ the eating of chocolate, her desirability values are $\mathcal{D}(C \& M)=-9, \mathcal{D}(\sim C \& M)=-10, \mathcal{D}(C \& \sim M)=1$, $\mathcal{D}(\sim C \& \sim M)=0$. She knows that eating chocolate does not cause migraines, but she knows that certain pre-migraine states dispose her to eat chocolate. This works via a mechanism to which she has no conscious access; she can not 'feel' a craving for chocolate. Thus when she finds herself eating chocolate, this provides her with some evidence that an unpleasant migraine is coming on. She now must choose whether or not to eat some chocolate. Since $P(C \square \rightarrow M)=P(\sim C \square \rightarrow M)$, causal decision theory recommends eating the chocolate: it is a harmless pleasure. However, the conditional probabilities $P(\mathrm{M} \mid C)$ and $P(\mathrm{M} \mid \sim C)$ will be different, and if the difference is large enough, EDT will recommend against eating chocolate. This recommendation can't be overturned by thinking more carefully about the nature of the choices that are available. Here, EDT seems to recommend acting so as to avoid receiving bad news.

A number of authors have defended EDT against these kinds of objections, and/or criticized CDT. I think both kinds of arguments are mostly mistaken, but will not attempt any defense of this belief here. My argument, I think, relies only on fairly weak assumptions about causal decision theory. First, I assume that its 
recommendations are extensionally correct in a broad range of ordinary cases. Second, I assume that there is something right about its guiding intuition, in the sense that there is some kind of important conceptual connection between causation and rational decision-making. Some authors, such as Eells (1982), Jeffrey (1983), Horwich (1987), and Price (1986), have argued that it is possible to formulate a version of EDT that replicates the intuitively correct recommendations of CDT. This is preferable to CDT, they claim, either because causation is a murky notion that it is best to avoid if possible, or else because it leaves open the possibility of analyzing causation in terms of decision, rather than vice versa. Horwich (1987) and Price (1991) explicitly adopt the latter strategy. This kind of program would not undermine my two assumptions. While it may be possible to formulate a causally innocent version of decision theory, CDT still gives the correct recommendations. And if causation is to be analyzed in terms of decision, there is an important conceptual link between them, even if CDT gets direction of the link backwards.

Others have maintained that CDT yields the wrong recommendations in certain extraordinary kinds of cases, such as Newcomb problems involving infallible predictors (Plantinga (1986)), or cases involving time travel (Egan 2007, 2009). ${ }^{9}$ Even if one accepts these counterexamples, CDT provides the correct recommendations in a broad range of ordinary cases. In all of these cases, CDT's recommendation to act so as to cause desirable outcomes is sound.

\section{CDT, Preemption and Overdetermination}

The guiding idea behind CDT is that one should act in such a way that one's actions cause desirable outcomes. But the standard formulation of CDT given in the previous section makes no explicit reference to causation. Rather, it requires the agent to reason using her degrees of belief about certain non-backtracking counterfactuals. Indeed, even David Lewis's version of CDT, published in 1981, makes no use of his own counterfactual analysis of causation. ${ }^{10}$ This leads to a very natural prediction: we should be able to construct counterexamples to CDT involving preemption or overdetermination.

But attempts to construct such counterexamples are frustrated. Suppose, for example, that Suzy desires that the bottle be shattered, and that she has no preference for throwing or not-throwing considered intrinsically. I.e. if $T$ says that Suzy throws her rock, and $S$ that the bottle shatters, her desirabilities satisfy $\mathcal{D}(T \& S)=\mathcal{D}(\sim T \& S)>\mathcal{D}(T \& \sim S)=\mathcal{D}(\sim T \& \sim S)$. Suppose also that if Suzy throws, her rock will shatter the bottle, and that if she doesn't throw, the bottle will still be shattered by Billy's rock. Finally, suppose that Suzy knows all of this (or at least believes it with degree of belief 1). Then CDT tells her that she should be indifferent between throwing her rock and not throwing. But this clearly

\footnotetext{
9 Arntzenius (2008) and Egan (2007) have argued that CDT yields the wrong conclusions in less outré cases. I think Joyce (2012) provides a convincing response.

${ }^{10}$ Lewis (1981) does cite his earlier paper (Lewis 1973), but only in the context of claiming that counterfactuals should not backtrack.
} 
is the right recommendation. If all she cares about is whether the bottle will shatter, and it will shatter no matter which action she chooses, then she should be indifferent. The fact that Suzy's throw would count as a cause of the bottle's shattering, despite the lack of counterfactual dependence, is irrelevant to her deliberations.

The case is slightly more complicated if Suzy is not certain that Billy's rock will smash the bottle if she does not throw. Consider first cases of symmetric overdetermination and late preemption, where both Suzy and Billy will throw. In this case, if Suzy is uncertain that Billy's throw will shatter the bottle, CDT might well recommend that she throw, in order to give an extra chance of success. In such a case, CDT would recommend throwing because Suzy thinks it's possible that the shattering of the bottle (or at least the probability of its shattering) is counterfactually dependent on her throw. And once again, it seems that CDT gives the right recommendation.

Finally, consider a case of early preemption, where Billy will throw only if Suzy doesn't. If Suzy is not certain that Billy's throw will hit the bottle, CDT will recommend that Suzy throw just in case she thinks her throw is more likely to smash the bottle than Billy's throw.

In all cases, CDT seems to give the right recommendation, despite the fact that it considers only counterfactual relations, and not causation per se. And it does so even in the very cases where we most expect causation and counterfactual dependence to come apart.

\section{Does Causal Decision Theory Deserve the Name?}

One reaction would be to claim that CDT is misnamed. It should really be called 'non-backtracking counterfactual dependence decision theory', although that is quite a mouthful. Since CDT is not defined in terms of genuinely causal relations, it does not deserve the epithet 'causal'.

I do not think that this is the correct diagnosis. Recalling the example of the barometer and the storm, we might ask: What is so special about causal relationships? Why do we not just make do with correlations? The answer, I think, is that if we are only interested in passive prediction, correlations will serve as well as genuinely causal relationships. However, as agents, we need knowledge of causal relationships to guide our interactions with the world. In the apt phrase of Nancy Cartwright (1979), causal relationships underwrite 'effective strategies'. Mellor (1995) takes the claim that causes are means for bringing about their effects to be a central 'connotation' of the concept of causation, and interprets this claim in broadly decision-theoretic terms. This key idea is also at the center of Woodward's (2003) interventionist account of causation: causal relationships are those that persist under interventions. Even if one does not agree with the specific theory of causation offered by any of these authors, it seems to me that they have provided the right answer to the question of what is distinctive and important about causal relations, in contrast, say, with mere correlations. Indeed, if one severs the conceptual link between causation and rational deliberation about action, it is hard to understand 
why we value knowledge of causal relations in the way we do. For example, why is medical research focused on evaluating causal claims, if not to guide treatment and prevention? So it seems to me that the sort of conceptual connection between causation and rational deliberation that guides causal decision theory is as central to the concept of causation as any intuition that threatens to sever this connection.

I will however, make one small concession to the objection. There are some cases where the counterfactual dependence that figures in CDT does not correspond to any kind of causal relation. This is when the antecedent and consequent of a counterfactual are not distinct, in the sense articulated by Lewis (1986a). For example, suppose that the agent values doing action $A$ for its own sake. Then a counterfactual such as $A \square \rightarrow A$, or perhaps $A \square \rightarrow A \& O$ will figure in her decision-theoretic calculations. In this case, the relation is not causal, but logical. Analogously, if the agent wants to write 'HELLO', and she is deliberating about whether to write 'ELL', the relation of counterfactual dependence will reflect the mereological relationship between these events, rather than causal dependence. Sometimes when we act, our action is not a means to some end, but rather an end in itself, or at least the partial fulfillment of some end. This last qualification will be important in Sect. 10 below.

\section{Actual Causation and Causal Dependence}

I wish to propose an alternate diagnosis. The specific relation that David Lewis and others who have been concerned with preemption and overdetermination cases have been trying to analyze is actual causation. It has been called by other names: 'singular causation', 'token causation', 'event causation', and just plain 'causation'. All of these names are misleading. 'Causation' is misleading because the particular relation does not exhaust the subject matter of causation. In particular, the relation that figures in formulations of causal decision theory is genuinely causal, but it is not actual causation.

It has been common to distinguish between 'singular', 'token', or 'event' causation, on the one hand, and 'general', 'type', or 'property' causation or 'causal laws', on the other. For example, in "Causation" Lewis (1973) writes:

1. I shall confine myself to causation among events...: flashes, battles, conversations, impacts, strolls, deaths, touchdowns, falls, kisses and the like. Not that events are the only things that can cause or be caused....

2. My analysis is meant to apply to causation in particular cases. It is not an analysis of causal generalizations. (Cited in Lewis 1986b, 161-2)

[See also, e.g., Sober (1985), Cartwright (1989, Chapter 3) and Eells (1991, Introduction and Chapter 6) for related distinctions.] This might suggest a way out. While Lewis and others have been trying to analyze singular causation or event causation, perhaps it is general causation or type causation that is relevant to decision theory.

But this is not quite right either. Suzy is deliberating about whether to throw this particular rock at this particular bottle on this particular occasion. In evaluating the 
desirability of throwing the rock, she wants to know what the effects of that particular throw will be. Suzy is not interested in whether throws in general cause bottle shatterings, except insofar as it is relevant to whether this particular throw will. So the relation that plays a role in decision theory is not general causation, or anything like that.

In "Causation," David Lewis introduces the term causal dependence to refer to non-backtracking counterfactual dependence among propositions describing the occurrence of events:

To any possible event $e$, there corresponds the proposition $E$ that holds at all and only those worlds where $e$ occurs. This $E$ is the proposition that $e$ occurs....

Let $c_{1}, c_{2}, \ldots$ and $e_{1}, e_{2}, \ldots$ be distinct possible events such that no two of the $c$ 's and no two of the $e$ 's are compossible. ${ }^{11}$ Then I say that the family $e_{1}$, $e_{2}, \ldots$ of events depends causally on the family $c_{1}, c_{2}, \ldots$ iff the family $E_{1}$, $E_{2}, \ldots$ of propositions depends counterfactually on the family $C_{1}, C_{2}, \ldots$. As we say it: whether $e_{1}$ or $e_{2}$ or... occurs depends on whether $c_{1}$ or $c_{2}$ or...occurs. ${ }^{12}$ (Cited from Lewis 1986b, 166; I have changed the notation slightly to maintain consistency)

What I want to suggest is that Lewis's relation of causal dependence is deserving of the epithet 'causal', not just because it plays a role in Lewis's ultimate definition of causation, nor because Lewis takes it to be sufficient (but not necessary) for causation, but rather because it is a genuine causal notion in its own right. It is just this notion of causal dependence that is captured in expressions of the form " $A_{i}$ $\square \rightarrow O_{j}{ }^{\prime}$ that figure in the formulation of CDT. ${ }^{13}$

The last claim requires three caveats. The first is that it is the agent's beliefs about the counterfactuals ' $A_{i} \square \rightarrow O_{j}$ ' that figure in CDT, rather than their truth values. The other two caveats are already stated by Lewis. If whether $o_{1}$ or $o_{2}$ or... occurs does not depend on whether the agent performs $a_{1}$ or $a_{2}$ or..., then counterfactuals of the form ' $A_{i} \square \rightarrow O_{j}$ ' will capture causal dependence only in a negative way: they will capture the fact that the outcome is not causally dependent upon the agent's action. The final caveat is that counterfactual dependence of $O_{j}$ on $A_{i}$ corresponds to causal dependence only in the case where $o_{j}$ is distinct from $a_{i}$.

So we have two causal notions in play here: actual causation, and causal dependence. ${ }^{14}$ Aside from the fact that the two notions come apart in cases of preemption and overdetermination, what can be said about the relationship between these two notions, or about the difference between them? I can't provide a complete

\footnotetext{
11 I would weaken the requirement on the $e$ 's to allow some duplication, so long as at least two of them are incompatible.

12 I would add that the probability that $e_{1}$ or $e_{2}$ or...occurs could depend on whether $c_{1}$ or $c_{2}$ or...occurs.

13 My position here is very similar to that of Hall (2004). Hall distinguishes two causal notions, 'production' and 'dependence'. Dependence is essentially causal dependence, as Lewis defines it. Hall (2004, 268-70) argues that it is dependence, not production, that figures in decision theory.

14 I do not at all intend that these are the only two causal notions. See Hitchcock (2007) for further discussion.
} 
answer, but I think that part of the answer is that actual causation is a retrospective notion, while causal dependence is a prospective notion.

Judgments of actual causation are typically expressed in the past tense. Some event has occurred, and we reason backward from this event to make a claim about the event's causes. The relation is called 'actual causation' (I believe first by Pearl 2000) because it depends upon how events in the world actually play out. This information is typically much easier to come by in hindsight than in foresight. For example, after Suzy has thrown her rock, and her rock has beaten Billy's to the bottle, we can look back and see that it was in fact Suzy's throw that caused the bottle to shatter. This dependence on the actual course of events is reflected in the fact that, in order to assess actual causation, we often have to make use of ENF counterfactuals. For example, when Suzy's throw preempts Billy's throw, we evaluate the claim that Suzy's throw caused the bottle to shatter, by considering the case where Suzy does not throw, but where we also hold fixed that Billy did not throw (or that his rock did not hit the bottle). Looking back, after the fact, we see that Billy did not in fact throw. We tend to think of the past as fixed, and hence it is natural to hold this fact fixed when evaluating the relevant counterfactual. By contrast, when Suzy is deliberating about whether or not to throw, it is still open whether or not Billy will throw. Indeed, it is Suzy's choice, which she has not yet made, that will determine whether Billy throws or not. It makes little sense for her to hold fixed that Billy will not throw when this has not yet been settled.

Because of its retrospective character, actual causation comes into play when some event has occurred, and we wish to assign responsibility for its occurrence. This happens when evaluating moral or legal responsibility for example, and also when explaining the occurrence of a particular event. It is, in part, because of its involvement with these topics of perennial philosophical interest that actual causation has received so much attention from philosophers.

By contrast, causal dependence is a largely prospective notion. It involves thinking forward from the occurrence or non-occurrence of some event, or more generally, from the occurrence of one or another from among a class of alternative events, or from the occurrence of some combination of events. It involves a consideration not just of what actually happens, but of what possibly could happen. In the framework of structural equations modeling (e.g. Pearl 2000), relations of causal dependence are represented by structural equations. Such equations represent the way in which the value of an effect variable would respond to all possible settings of the values of its immediate causes.

The context of decision theory is prospective. One is faced with a choice of possible actions. One evaluates an action by reasoning forward from the action to all of its various effects. For this reason, CDT is built upon the notion of causal dependence, rather than upon actual causation.

It is interesting to note that there are no decision-theoretic analogs to the problem of moral luck, or the principle of double effect. If an agent chooses an action with a lower expected payoff and lucks out, no one thinks that the agent was rational or prudent as a result of this good fortune. Likewise, no one thinks it is irrational to do something mildly unpleasant as a means of achieving a highly desired end. I suspect that these differences between morality, on the one hand, and prudential rationality, 
on the other, result from the retrospective nature of moral evaluation, in contrast with the prospective nature of rational deliberation.

Note also that by 'prospective', I do not just mean 'in the future', but also something like 'forward from a cause'. Likewise, 'retrospective' means something like 'backward from an effect'. There may be interesting hybrid cases. For example, suppose I want to achieve a specific objective $O$ at time $t_{0}$. I reason backward from this goal, deciding first what I must do at time $t_{-1}$. Then I reason back further, deciding what I must do at $t_{-2}$, given what I will later be doing at $t_{-1}$, and so on. This kind of reasoning process will involve ENF counterfactuals, and hence it may employ a causal notion that more closely resembles actual causation than causal dependence.

\section{Barking up the Wrong Tree?}

If this diagnosis is correct, it should go some way toward countering the worry, described in Sect. 3 above, that counterfactual approaches to causation are barking up the wrong tree. For suppose one accepts that there is a strong conceptual link between causation and rational deliberation, and that causal decision theory is right (at least in a broad range of cases) in tying rational choice to relations of causal dependence. Then it seems to follow that there is one important causal concept-causal dependencethat the simplest counterfactual theory of causation gets exactly right. So counterfactual theories of causation are not just barking up the wrong tree.

This is not to say that there is no important work to be done. We still do not have a fully adequate account of the relevant non-backtracking counterfactuals, nor do we have an adequate account of the precise relationship between actual causation and causal dependence. But it's nice to know that there is something of value up the tree at which one is barking.

\section{An Objection}

I have heard the following objection on several occasions: Consider again the case where Suzy is deliberating about whether to throw her stone at the bottle. In my discussion of this case in Sect. 6 above, I assumed that Suzy only cares about whether or not the bottle shatters. But why restrict things in this way? Surely Suzy might well care whether she causes the bottle to shatter; i.e. about whether her action is an actual cause of the bottle's shattering. So perhaps CDT needs to incorporate relations of actual causation after all. [Ned Hall (2004) entertains a very similar objection, and responds along essentially the same lines as I do.]

Let us sharpen the objection. Suzy might care about whether she causes the bottle to shatter because she wants to receive the credit or avoid the blame for shattering it. This case requires no modification of CDT. For example, if Suzy will receive credit for throwing her rock and shattering the bottle, and she desires credit, over and above her intrinsic desire for seeing the bottle shattered, then we need to partition the outcomes more finely. Now the outcomes are not simply 'bottle shatters' and 
'bottle doesn't shatter', but something like 'bottle shatters and Suzy receives credit', 'bottle shatters and Suzy doesn't receive credit', and 'bottle doesn't shatter'. While the shattering of the bottle does not depend counterfactually on Suzy's throw, her receiving credit does so depend. So CDT, as formulated in terms of counterfactual dependence, would correctly recommend throwing. There is no need to inject actual causation into CDT to handle this kind of case.

So suppose instead that Suzy values being an actual cause of the bottle's shattering for its own sake. This can't be captured using an independent outcome, such as receiving credit, that is counterfactually dependent upon Suzy's action. Doesn't this show that an account of rational decision needs to take account of relations of actual causation?

I don't think that it does. Suzy might, in principle, care about anything. Perhaps Suzy loves ice cream. Let us suppose that many of Suzy's decisions center around ice cream. If she is out of ice cream at home, she will go out of her way to buy some on her way home from work. She always chooses to eat lunch at a particular cafeteria where ice cream is served. She plans vacations around great ice cream parlors around the world that she has read about on the internet. And so on. This is all possible. But it would in no way show that decision theory is really about ice cream, or that an adequate decision theory would have to make reference to ice cream. Of course, in order to represent a particular decision situation in which Suzy is deliberating about what kind of ice cream to eat, we will need a language in which we can represent her options: Suzy eats vanilla ice cream, Suzy eats pistachio ice cream, etc. But this does not mean that ice cream has to be built into the very structure of decision theory.

If Suzy happens to care about whether or not her action is an actual cause of the bottle's shattering, then in order to represent this decision situation, we will need to be able to represent the different possible outcomes: The bottle shatters and Suzy is an actual cause, the bottle shatters but Suzy is not an actual cause, etc. But what matters to the calculation is how the truth of these propositions depends counterfactually upon her action. In this case, the non-backtracking counterfactual dependence is not quite the same as causal dependence, because the antecedent and consequent of the counterfactual will not describe distinct events (in the sense required, e.g., by Lewis 1986a). This is the special case described in Sect. 7 above. But the fact that Mary could, in principle, care about whether her action is an actual cause of the bottle's shattering in no way shows that decision theory itself needs to be formulated in terms of actual causation, any more than the fact that Mary could care deeply about ice cream shows that decision theory needs to be formulated in terms of ice cream.

Acknowledgments For discussion, suggestions, and comments, I would like to thank Kenny Easwaran, Luke Glynn, Joel Velasco, audience members at the workshop on actual causation at the University of Konstanz, audience members at the University of California, San Diego, and two anonymous referees.

\section{References}

Albert, D. (2000). Time and chance. Cambridge, MA: Harvard University Press. 
Arntzenius, F. (2008). No regrets, or: Edith Piaf revamps decision theory. Erkenntnis, 68, 277-297.

Beebee, H., Hitchcock, C., \& Menzies, P. (2009). The Oxford handbook of causation. Oxford: Oxford University Press.

Cartwright, N. (1979). Causal laws and effective strategies. Noûs, 13, 419-437.

Cartwright, N. (1989). Nature's capacities and their measurement. Oxford: Oxford University Press.

Collins, J., Hall, N., \& Paul, L. (Eds.). (2004). Causation and counterfactuals. Cambridge MA: MIT Press.

Eells, Ellery. (1982). Rational decision and causality. Cambridge: Cambridge University Press.

Eells, E. (1991). Probabilistic causality. Cambridge: Cambridge University Press.

Egan, A. (2007). Some counterexamples to causal decision theory. Philosophical Review, 116, 93-114.

Egan, A. (2009) Time travel, decision theory, and respect for the actual, presented at the Kline Workshop, University of Missouri, September 2009.

Frankfurt, H. (1969). Alternate possibilities and moral responsibility. Journal of Philosophy, 66, 829-839.

Gibbard, A., \& Harper, W. (1978). Counterfactuals and two kinds of expected utility. In C. Hooker, J. Leach, \& E. McClennen (Eds.), Foundations and applications of decision theory (pp. 125-162). Dordrecht: Reidel.

Hall, N. 2004. Two concepts of causation. In Collins, Hall, \& Paul (Eds.), (pp. 225-276).

Halpern, J., \& Pearl, J. (2005). Causes and explanations: A structural-model approach—Part I: Causes. British Journal for the Philosophy of Science, 56, 843-887.

Hitchcock, C. (2001). The intransitivity of causation revealed in equations and graphs. Journal of Philosophy, 98, 273-299.

Hitchcock, C. (2007). How to be a causal pluralist. In P. Machamer \& G. Wolters (Eds.), Thinking about causes: From Greek philosophy to modern physics (pp. 200-221). Pittsburgh: University of Pittsburgh Press.

Hobart, R. (1934). Free will as involving determinism and inconceivable without It. Mind, 43, 1-27.

Horwich, P. (1987). Asymmetries in time. Cambridge, MA: M.I.T. Press.

Hume, D. (1748). An enquiry concerning human understanding.

Jeffrey, R. (1969). Statistical Explanation vs. Statistical Inference. In N. Rescher (Ed.), Essays in honor of Carl G Hempel (pp. 104-113). Dordrecht: D. Reidel.

Jeffrey, R. (1983). The logic of decision (2nd ed.). Chicago: University of Chicago Press.

Joyce, J. (1999). The foundations of causal decision theory. Cambridge: Cambridge University Press.

Joyce, J. (2012). Regret and instability in causal decision theory. Synthese, 187, 123-145.

Kutach, D. (2003). The entropy theory of counterfactuals. Philosophy of Science, 69, 82-104.

Lewis, D. (1973). Causation. Journal of Philosophy, 70, 556-567. Reprinted in Lewis (1986b), pp. $159-172$.

Lewis, D. (1979). Counterfactual dependence and time's arrow. Nô̂s, 13, 455-476. Reprinted in Lewis (1986b), pp. 32-52.

Lewis, D. (1981). Causal decision theory. Australasian Journal of Philosophy, 59, 5-30. Reprinted in Lewis (1986b), pp. 305-337.

Lewis, D. (1986a). Events. In Lewis (1986b), pp. 241-270.

Lewis, D. (1986b). Philosophical papers (Vol. II). Oxford: Oxford University Press.

Lewis, D. (1997). Finkish dispositions. Philosophical Quarterly, 47, 143-158.

Lewis, D. (2000). Causation as influence. Journal of Philosophy, 97, 182-197.

Loewer, B. (2007). Counterfactuals and the second law. In Price, \& Corry (2007), (pp. 293-326).

Malcolm, N. (1963). Knowledge and certainty. Englewood Cliffs, NJ: Prentice-Hall.

Martin, C. B. (1994). Dispositions and conditionals. Philosophical Quarterly, 44, 1-8.

Maudlin, T. (2004). Causation, counterfactuals, and the third factor. In Collins, Hall, \& Paul (2004), pp. 419-443. Reprinted in Maudlin (2007).

Maudlin, T. (2007). The metaphysics within physics. Oxford: Oxford University Press.

Mellor, D. H. (1995). The facts of causation. London: Routledge.

Moore, M. (2009). Causation and responsibility: An essay in law, morals, and metaphysics. Oxford: Oxford University Press.

Munsat, S. (1966). The concept of memory. New York: Random House.

Paul, L. (2009). Counterfactual theories of causation. In Beebee, Hitchcock, \& Menzies (2009), (pp. $158-184)$.

Pearl, J. (2000). Causality: Models, reasoning, and inference. Cambridge: Cambridge University Press.

Plantinga, A. (1986). Ockham's way out. Faith and Philosophy, 3, 256.

Price, H. (1986). Against causal decision theory. Synthese, 67, 195-212. 
Price, H. (1991). Agency and probabilistic causality. British Journal for the Philosophy of Science, 42, $15-76$.

Price, H., \& Corry, R. (2007). Causation, physics, and the constitution of reality. Oxford: Oxford University Press.

Shope, R. (1978). The conditional fallacy in contemporary philosophy. Journal of Philosophy, 75, 397-413.

Smith, M. (2003). Rational Capacities, or: How to distinguish recklessness, weakness, and compulsion. In S. Stroud, \& C. Tappolet (Eds.), Weakness of will and practical irrationality.

Sober, E. (1985). Two concepts of cause. In Peter Asquith \& Philip Kitcher (Eds.), PSA 1984 (Vol. II, pp. 405-424). Philosophy of Science Association: East Lansing.

Sosa, E., \& Tooley, M. (Eds.). (1993). Causation. Oxford: Oxford University Press.

Woodward, J. (2003). Making things happen: A theory of causal explanation. Oxford: Oxford University Press.

Yablo, S. (2002). De Facto dependence. Journal of Philosophy, 99, 130-148. 